\title{
Electrodynamic Tether at Jupiter-II: Fast Moon Tour After Capture
}

\author{
Juan R. Sanmartin, Mario Charro, Enrico C. Lorenzini, Henry B. Garrett, \\ Claudio Bombardelli, and Cristina Bramanti
}

\begin{abstract}
An electrodynamic bare-tether mission to Jupiter, following the capture of a spacecraft (SC) into an equatorial highly elliptical orbit with perijove at about 1.3 times the Jovian radius, is discussed. Repeated applications of the propellantless Lorentz drag on a spinning tether, at the perijove vicinity, can progressively lower the apojove at constant perijove, for a tour of Galilean moons. Electrical energy is generated and stored as the $\mathrm{SC}$ moves from an orbit at $1: 1$ resonance with a moon, down to resonance with the next moon; switching tether current off, stored power is then used as the SC makes a number of flybys of each moon. Radiation dose is calculated throughout the mission, during capture, flybys and moves between moons. The tour mission is limited by both power needs and accumulated dose. The three-stage apojove lowering down to Ganymede, Io, and Europa resonances would total less than 14 weeks, while 4 Ganymede, 20 Europa, and 16 Io flybys would add up to 18 weeks, with the entire mission taking just over seven months and the accumulated radiation dose keeping under $3 \mathrm{Mrad}$ (Si) at 10-mm $\mathrm{Al}$ shield thickness.
\end{abstract}

\section{INTRODUCTION}

$\mathbf{T}$ HE JOVIAN mission discussed here involves an electrodynamic bare tether collecting orbital-motion-limited (OML) current from the ambient plasma to tap Jupiter's rotational energy for both propulsion and power. The (circular, equatorial) stationary orbit is close to Jupiter, at radius $a_{s} \approx$ $2.24 R_{J}$, because of both fast rotation and low density of the planet; the large Jovian magnetic field $\bar{B}$ then allows plasma to corotate with the planet also beyond that orbit. The positions of perijove and apojove in elliptical prograde orbits relative to the stationary orbit, which lies at an energy maximum in the orbit/planet-spin interaction, might be exploited to conveniently make the induced Lorentz force to be drag or thrust while generating power and navigating the system. The dense-plasma Io torus, lying well beyond the stationary orbit, might allow thrusting, although $B$ would be weak [1].

The capture operation was analyzed in previous work [2]. Design parameters such as (tape) tether length $L$ and thickness $h$ and perijove radius $r_{p}$ faced opposite constraints. Capturing a full spacecraft (SC) mass $M_{\mathrm{SC}}$ several times its tether mass $m_{t}$ requires a low perijove and a high $L^{3 / 2} / h$ ratio. However, tether bowing and tensile stress considerations arising from the tether spin required by the low-gravity gradients and high lateral Lorentz forces at Jupiter place a bound on the $L^{5 / 2} / h$ ratio. Also, maximum tether temperature scales as $L^{3 / 8}$. In addition, both tether temperature and bowing are greater when the perijove is closer to Jupiter.

Capture required an electrodynamic drag work $W_{c}$ such that $\left|W_{c}\right|$ exceeds the positive energy in the incoming hyperbolic orbit. The greater that work, the lower the apojove radius and eccentricity in the first orbit following capture. In a preliminary design, a reinforce $\mathrm{Al}$ tape that is $50 \mathrm{~km}$ long and $0.05 \mathrm{~mm}$ thick, coated for thermal emittance of about 0.8 and spinning with 12-min period, fulfills the following: 1) satisfies all constraints and 2) captures a SC with full mass over three times $m_{t}$ at a $1.3 R_{J}$ perijove, with $\left|W_{C}\right|$ being twice the excess hyperbolic energy of a Hohmann transfer. We will use this tape as reference tether for numerical considerations. With both cross-sectional area and perimeter of a thin tape of given thickness being proportional to its width $w$, no characteristic dimensionless number involves $w$; the SC mass will just scale up with $w$ in a range allowing from about 0.2 to $5 \mathrm{t}$. We will set $w=3 \mathrm{~cm}$ wherever choosing a definite mass is convenient, with $m_{t}$ being $202 \mathrm{~kg}$ and $M_{\mathrm{SC}}$ being about $650 \mathrm{~kg}$ [2].

In this paper, we analyze closed-orbit evolution after capture, for a Galilean moon tour under repeated Lorentz forces, which requires no propellant and no independent power supply; alternative missions after capture (acquiring low circular orbits around Jupiter and around moon Io) will be analyzed in work to follow. If current is on along the arc where the Lorentz force is drag, as the SC nears perijove, the apojove radius $r_{a}$ will be reduced. Further reductions will occur at successive perijove passes, resulting in a series of elliptical orbits with common perijove and decreasing eccentricities, with changes in the perijove position being small second-order effects.

With the Lorentz force only acting around perijove, the energy per unit mass $\varepsilon$ of the incoming hyperbolic orbit and 
following elliptical orbits only depends on eccentricity $e$ through a standard formula in orbital mechanics [2]

$$
\varepsilon=\frac{-\mu_{J}}{2 r_{p}}(1-e)
$$

where $\mu_{J}$ is Jupiter's gravitational parameter. We assume that the hyperbolic eccentricity, $e_{h}=1+r_{p} v_{\infty}^{2} / \mu_{J}$, is just above unity. Indeed, in the Hohmann transfer case $\left(v_{\infty} \approx 5.64 \mathrm{~km} / \mathrm{s}\right)$, we have $e_{h}-1 \approx 0.018 r_{p} / R_{J}$. Also, as we will recall, the $\mathrm{S} / \mathrm{C}$ can be barely captured; this leads to an energy balance, using (1)

$$
\frac{-W_{c}}{M_{\mathrm{SC}} v_{\infty}^{2} / 2}=\frac{-\Delta \varepsilon}{v_{\infty}^{2} / 2}=\frac{-\Delta e}{e_{h}-1}=O(1) .
$$

The orbit is thus hardly affected locally, allowing to approximate it as parabolic $(e=1)$ throughout capture. As the eccentricity decrement in the following perijove passes will be found to be also small, calculations will be carried out here as if eccentricity, although different from unity, was kept constant during each pass. The decrement $\Delta e$ at fixed $e$ is given by an equation similar to (2)

$$
\frac{2 W_{d, e}}{m_{t} v_{\infty}^{2}}=\frac{M_{\mathrm{SC}}}{m_{t}} \frac{\Delta e}{e_{h}-1}
$$

where $W_{d, e}$ is the drag work.

Propulsive performance is dependent on orbit geometry, as well as on ambient conditions, namely, electron plasma density $N_{e}$, field $\bar{B}$, and motional electric field

$$
\bar{E}_{m}=\bar{v}^{\prime} \times \bar{B}
$$

where $\bar{v}^{\prime} \equiv \bar{v}-\bar{v}_{\mathrm{pl}}$ is the SC velocity relative to the corotating plasma, which is perpendicular to $\bar{B}$. A simple no-offset no-tilt dipole magnetic model

$$
B=B_{s} a_{s}^{3} / r^{3}\left(B_{s} \approx 0.38 \mathrm{G}\right)
$$

will do in our analysis [3]. We shall use the Divine-Garrett model of the thermal Jovian plasma in the plasmasphere, which is then longitude independent; only the plasma density profile, which has a simple analytical representation, will be involved in the calculations [4].

Lowering the orbit apojove through a series of perijove passes is considered in Section II. In Section III, we discuss using the tether to serve as its own power source by having an electric load plugged in; a large energy could be tapped (used locally or stored for later use) from the big power developed during SC capture and following high-current operations, with negligible effect on its dynamics. Radiation dose through the successive orbits places a limit on postcapture operation, with the GIRE radiation model being used in Section IV to evaluate the accumulated dose [5]. A tour of Galilean moons is designed in Section V.

\section{LOWERING THE APOJOVE}

Calculating the drag work $W_{d, e}$ follows closely the analysis for capture work in [2]. The Lorentz force on a bare tether involves the length-averaged current $I_{\mathrm{av}}$, which depends on impedances in the tether circuit. The tether would spin in the equatorial orbital plane, perpendicular to the magnetic field, with state-of-the-art hollow cathodes at both ends taking active turns as each end becomes cathodic; typically, they only require tens-of-volt bias, making for negligible contact impedance. We shall also neglect both the radiation impedance for current closure in the Jovian plasma (indeed negligible at Earth) and any power-output impedance, which is discussed in the next section. The average tether current will then lie between extreme values, with one corresponding to no ohmic effects and the other to ohmic-limited (short-circuit) current.

The power of the magnetic (Lorentz) force $\bar{F}_{M}$ for a general elliptical orbit reads

$$
\dot{W}=\bar{v} \cdot \bar{F}_{M}=v \bar{u}_{t} \cdot\left(-I_{\mathrm{av}} \bar{u} \times L B \bar{k}\right)
$$

where $\bar{u}_{t}, \bar{u}$, and $\bar{k}$ are unit vectors along SC velocity, tether line from cathode to anode, and magnetic field (pointing south at the equator, $\bar{B}=-B \bar{k}$ ), respectively. The current is normalized with the short-circuit current as maximum possible value

$$
I_{\mathrm{av}} / \sigma_{c} w h E_{m} \equiv i_{\mathrm{av}} \leq 1
$$

where $\sigma_{c}$ is the tether conductivity and $E_{m}$ is the projection of the field $\bar{E}_{m}$ along the tether

$$
E_{m} \equiv \bar{E}_{m} \cdot \bar{u}=v^{\prime} B \cos \varphi
$$

with $\varphi$ being the angle between tether unit vector $\bar{u}$ and $\bar{E}_{m}$. We can now write

$$
\left(\bar{u} \times \bar{u}_{t}\right) \cdot \bar{k}=-\sin \left(\varphi+\alpha_{E}\right)
$$

where $\alpha_{E}$ is the angle between $\bar{E}_{m}$ and the velocity $\bar{v}$ along $\bar{u}_{t}$. Averaging over angle $\varphi$ at fixed SC position gives

$$
\langle\dot{W}\rangle=-\sigma_{c} w h L B^{2} v v^{\prime} \sin \alpha_{E}\left\langle i_{\mathrm{av}} \cos ^{2} \varphi\right\rangle
$$

where we used the vanishing of the average $\left\langle i_{\mathrm{av}} \cos \varphi \sin \varphi\right\rangle$, arising from both (6) and the dependence $i_{\mathrm{av}} \equiv i_{\mathrm{av}}\left(E_{m}\right)$ recalled hereinafter. Since $\bar{v}^{\prime}$ and $\bar{E}_{m}$ are perpendicular to each other, we can write

$$
v v^{\prime} \sin \alpha_{E}=v \bar{v}^{\prime} \cdot \bar{u}_{t}=\bar{v} \cdot\left(\bar{v}-\Omega_{J} r \bar{u}_{\theta}\right)=v^{2}-\Omega_{J} r_{p} v_{p}
$$

in (7). We used corotation velocity $\bar{v}_{\mathrm{pl}}=\Omega_{J} r \bar{u}_{\theta}$ with planetary spin $\Omega_{J}=\sqrt{ } \mu_{J} / a_{s}^{3}$, and conservation of angular momentum $r \bar{v} \cdot \bar{u}_{\theta}=r_{p} v_{p}$, where $\theta$ and $\bar{u}_{\theta}$ are the true anomaly and transverse unit vector, respectively.

We next integrate the power in (7) over the time $\Delta t$ in the drag arc

$$
W_{d, e}=\int_{\Delta t}\langle\dot{W}\rangle d t=2 \int_{r_{p}}^{r_{u}} \frac{\langle\dot{W}\rangle d r}{d r / d t}
$$

taking $v^{2}$ in (8) from the energy equation for an elliptical orbit

$$
v^{2}=\mu_{J}\left(\frac{2}{r}-\frac{1-e}{r_{p}}\right)
$$


and using $r^{2} d \theta / d t=r_{p} v_{p}$ and the orbit equation $1+e \cos \theta=$ $(1+e) r_{p} / r$ in determining $d r / d t$. For a range of eccentricities below 1, the upper end at the integral $r_{u}$ marks the limit of the drag arc, where the tangential component of the relative velocity $v_{t}^{\prime}$ vanishes; as eccentricity decreases, however, a value is reached such that $r_{u}$ is the apojove radius $r_{a} \equiv r_{p}(1+$ $e) /(1-e)$.

Using (9) in (3), we finally find the decrement $\Delta e$ per orbit

$$
\begin{aligned}
\frac{-\Delta e}{e_{h}-1}= & \frac{m_{t}}{M_{\mathrm{SC}}} \widetilde{B}_{s}^{2} S_{e}\left(\frac{r_{p}}{R_{J}}, \Lambda, e\right) \\
S_{e} \equiv & \widetilde{r}_{M}^{8 / 3} \int_{1}^{\widetilde{r}_{u}} \frac{d \widetilde{r}}{\widetilde{r}^{6}} \frac{2 \widetilde{r}_{M}-\widetilde{r}\left[\sqrt{2(1+e)}+(1-e) \widetilde{r}_{M}\right]}{\sqrt{2(\widetilde{r}-1)} \sqrt{1+e-(1-e) \widetilde{r}}} \\
& \times\left\langle 2 i_{\mathrm{av}} \cos ^{2} \varphi\right\rangle \\
\widetilde{B}_{s}^{2} \equiv & \frac{\sigma_{c} B_{s}^{2} a_{s} v_{s 1}}{2^{5 / 6} \rho_{t} v_{\infty}^{2}} \approx 2.11
\end{aligned}
$$

for an aluminum tape and Hohmann transfer. In the previous, we wrote for convenience

$$
\begin{gathered}
\widetilde{r} \equiv \frac{r}{r_{p}} \\
r_{M}\left(r_{p}\right) \equiv a_{s} \sqrt{2 a_{s} / r_{p}}
\end{gathered}
$$

and used the parabolic velocity at $a_{s}$ as the reference velocity, $v_{s 1} \equiv \sqrt{2 \mu_{J} / a_{s}} \approx 39.8 \mathrm{~km} / \mathrm{s}$. Equation (11) gives the eccentricity decrement, normalized with the hyperbolic eccentricity excess $\left(e_{h}-1\right)$, as proportional to the tether-to-SC-mass ratio and the characteristic dimensionless parameter $\widetilde{B}_{s}^{2}$, and to a function $S_{e}$ given by (12), which is, in turn, dependent on orbit data $e$ and $r_{p}$ (or $r_{M}$ ), and an ambient parameter $\Lambda$ through the dimensionless average current $i_{\mathrm{av}}$. Note that capture requires a minimum decrement $|\Delta e|=e_{h}-1$, which is proportional to $v_{\infty}^{2}$, with the corresponding mass ratio then depending on the hyperbolic excess velocity, too; on the other hand, given the mass ratio, $\Delta e \propto\left(e_{h}-1\right) \widetilde{B}_{s}^{2}$ is independent of $v_{\infty}$.

The current $i_{\text {av }}$ is a universal function $i_{\text {av }}(\hat{L})$ given in [2]. In the case of negligible ohmic effects, bare-tether analysis shows the tether to be biased positive throughout its length, and the average current to be two-fifths of the OML current collected by the tether if at uniform bias $E_{m} L$

$$
I_{\mathrm{av}}(\mathrm{OML})=\frac{2}{5} \frac{2 w L}{\pi} e N_{e} \sqrt{\frac{2 e E_{m} L}{m_{e}}} .
$$

In general, $i_{\mathrm{av}}$ is found to be a function of the ratio $I_{\text {av }}(\mathrm{OML}) / \sigma_{c} w h E_{m} \equiv 3 \hat{L}^{3 / 2} / 10$, with $\hat{L}$ as given in [2]

$$
\begin{aligned}
& \hat{L}\left(\cos \varphi, \widetilde{r}, e, \Lambda, \widetilde{r}_{M}\right) \\
& \quad \equiv \frac{\Lambda \widetilde{r}^{7 / 6} /|\cos \varphi|^{1 / 3} \widetilde{r}_{M}^{4 / 9}}{\left[\widetilde{r}_{M}^{2}+\widetilde{r}^{3}-\left(\sqrt{2(1+e)}+\frac{1-e}{2} \widetilde{r}_{M}\right) \widetilde{r}_{M} \widetilde{r}\right]^{1 / 6}}\left(\frac{N_{e}}{N_{s}}\right)^{2 / 3}
\end{aligned}
$$

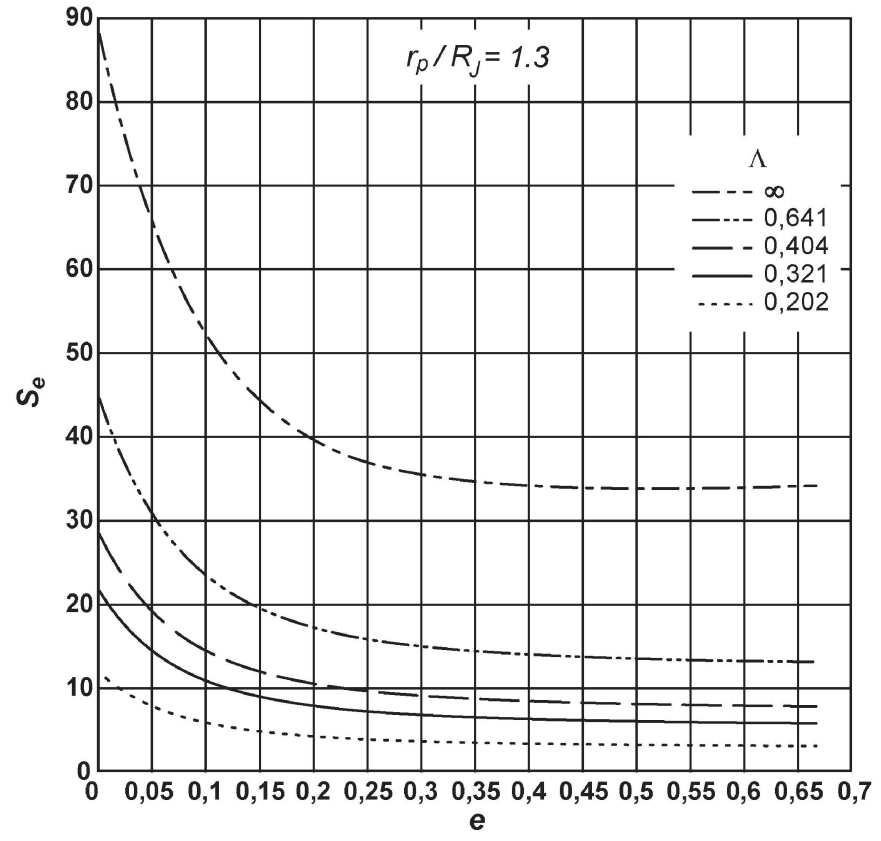

Fig. 1. Factor $S_{e}$ in (11) for drag work per orbit versus orbit eccentricity $e$, for $r_{p}=1.3 R_{J}$ and several values of parameter $\Lambda$, given in (17).

$$
\begin{aligned}
\Lambda & \equiv \frac{2^{49 / 18}}{(3 \pi)^{2 / 3}}\left(\frac{N_{s}}{\sigma_{c} h}\right)^{2 / 3} \frac{e L}{\left(m_{e} v_{s 1} B_{s}\right)^{1 / 3}} \\
& \approx 0.200 \frac{L}{50 \mathrm{~km}}\left(\frac{0.05 \mathrm{~mm}}{h}\right)^{2 / 3}
\end{aligned}
$$

where we used the conductivity of aluminum, the (no-tilt) Divine-Garrett density model at the equator, and the density $N_{s}=1.44 \times 10^{2} \mathrm{~cm}^{-3}$ at $a_{s}[4]$

$$
\begin{aligned}
N_{e}=\frac{4.65}{\mathrm{~cm}^{3}} & \exp \left(\frac{7.68 R_{J}}{r}\right) \\
& \Rightarrow \quad \frac{N_{e}}{N_{s}}\left(\widetilde{r}, \widetilde{r}_{M}\right)=\exp \left(2.2 \frac{\widetilde{r}_{M}^{2 / 3}}{\widetilde{r}}-3.43\right)
\end{aligned}
$$

Fig. 1, showing $S_{e}$ versus $e$ for $r_{p}=1.3 R_{J}$ and several $\Lambda$ values, proves $S_{e}$ to be nearly independent of eccentricity $\left(S_{e} \approx S_{1}\right.$ or $W_{d, e} \approx W_{c}$ ), except at small $e$. We note that drag acts over the entire orbit if $r_{a}<2.05 R_{J}$ for $r_{p}=1.3 R_{J}$, or $e<$ 0.22 , which falls in the eccentricity range showing a rapid increase of $S_{e}$ in Fig. 1; for $r_{p}=1.1 R_{J}$ and $r_{p}=1.5 R_{J}$, we find full-orbit drag for $e<0.29\left(r_{a}<2.0 R_{J}\right)$ and $e<0.17\left(r_{a}<\right.$ $2.1 R_{J}$ ), respectively. For the moon tour in Section V, which does not involve small $e$ values, we may therefore use $S_{e}=$ $S_{1}\left(r_{p} / R_{J}, \Lambda, 1\right)$ throughout in (11); $S_{1}$ is the function called $S\left(r_{p} / R_{J}, \Lambda\right)$ in [2].

Furthermore, the limit $\Lambda \rightarrow \infty$, with $\hat{L} \rightarrow \infty$ and $i_{\text {av }} \rightarrow 1$, which corresponds to dominant ohmic effects, is shown in (17) to require impracticably high values of the tape ratio $L / h^{3 / 2}$. Reasonably sized tapes, such as our reference design tape, make $\Lambda$ small and allow one to neglect ohmic effects, 


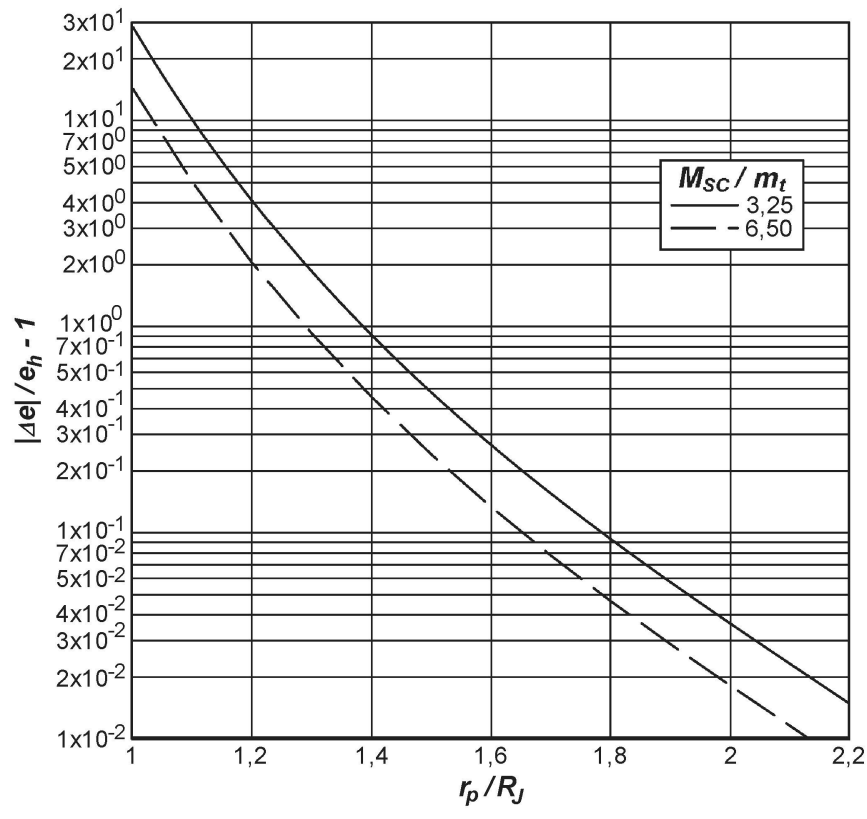

Fig. 2. Normalized eccentricity decrement per orbit versus perijove radius for a 50-km-long 0.05-mm-thick (small $\Lambda$, i.e., no ohmic effects) aluminum tape in Hohmann transfer for two values of the captured-mass ratio in (11a).

with $i_{\mathrm{av}} \approx I_{\mathrm{av}}(\mathrm{OML}) / \sigma_{c} E_{m} w h=0.3 \hat{L}^{3 ! 2}$ in $S\left(r_{p} / R_{J}, \Lambda\right)$, yielding

$$
\frac{-\Delta e}{e_{h}-1}=\frac{m_{t}}{M_{\mathrm{SC}}} \times 0.15\left(\frac{L}{50 \mathrm{~km}}\right)^{3 / 2} \frac{0.05 \mathrm{~mm}}{h} \Sigma\left(\frac{r_{p}}{R_{J}}\right)
$$

where we used (13). The function $\Sigma$, as given in [2], is used in Fig. 2 to represent the eccentricity decrement versus perijove radius for the reference 50-km-long 0.05-mm-thick tape, and two values of the mass ratio roughly corresponding to decrements $\Delta e=1-e_{h}$ and $2\left(1-e_{h}\right)$.

We can now readily describe orbit evolution in terms of the number of successive perijove passes. For Hohmann transfer and the 50-km-long $0.05-\mathrm{mm}$-thick aluminum reference tape with $M_{\mathrm{SC}}$ over three times $m_{t}$, and capture at $1.3 R_{J}$ perijove, we have $e_{h} \approx 1.02, e_{1} \approx 0.98$, and $\Delta e \approx-0.04$ at not too small $e$. A series of passes at fixed perijove, with repeated small decrements in eccentricity, would then result in a sequence of $e$ values, i.e., $0.98,0.94,0.90,0.86,0.82,0.78,0.74,0.70, \ldots$. The orbital period of the SC after each perijove pass is $\tau_{\text {orb }} \propto$ $\left[r_{p} /(1-e)\right]^{3 / 2}$, yielding a corresponding sequence of periods, i.e., $64.8,12.4,5.8,3.5,2.4,1.78,1.37,1.13, \ldots$ days. Note, however, that flyby operations to be discussed in Section V will modify that sequence.

\section{Power Budget}

During capture, a very large amount of energy would be taken from the orbital motion of the SC into the tether electric circuit, and ultimately transformed into thermal energy of the tether, to be radiated away, as discussed in [2]. From (2), with $\Delta e \approx-2\left(e_{h}-1\right)$, we have

$$
\frac{\left|W_{c}\right|}{M_{\mathrm{SC}}}=v_{\infty}^{2} \approx \frac{8.84 \mathrm{MW} \cdot \mathrm{h}}{10^{3} \mathrm{~kg}}
$$

or $5.75 \mathrm{MW} \cdot \mathrm{h}$ for the $650-\mathrm{kg} \mathrm{SC}$ corresponding to the 3 -cm-wide reference tape. Clearly, a small fraction of that energy could be taken by electric loads at the tether ends, with negligible effect on tether current and, thus, on the dynamics of capture. A small part of that energy could be used during capture, but a much greater part, $E_{\mathrm{st}}$, might be saved/stored in batteries or regenerative fuel cells for later use (for instance, for powering electrical propulsion if convenient). Similar results apply for each of the successive perijove passes.

The saved energy would be basically limited by the mass of the storage device. Storing a $0.5 \%$ energy fraction, or about $E_{\text {st }} \sim 30 \mathrm{~kW} \cdot \mathrm{h}$, could provide $250-\mathrm{W}$ power during $120 \mathrm{~h}$. The cycle life of the batteries, as considered in Section V, would be low, e.g., a few tens of cycles, possibly allowing the use of batteries with specific energy as high as $0.5 \mathrm{~kW} \cdot \mathrm{h} / \mathrm{kg}$, yielding a mass of $60 \mathrm{~kg}$. In the case of a regenerative fuel cell, both cell and fuel (hydrogen plus required oxygen) masses contribute to system mass. The ideal specific power of the fuel is about $4.3 \mathrm{~kW} \cdot \mathrm{h} / \mathrm{kg}$, but the masses of storage tanks and inefficiencies would make $2 \mathrm{~kW} \cdot \mathrm{h} / \mathrm{kg}$ a more realistic figure, yielding a fuel-related mass of $15 \mathrm{~kg}$. With cell specific power being on the order of $100 \mathrm{~W} / \mathrm{kg}$, the overall storage mass would be under $20 \mathrm{~kg}$. Fuel storage could be a main issue [6], [7].

Power decays rapidly away from its peak at the perijove, which is a result of the density profile being very steep near Jupiter, with most of the energy decrease $\left|W_{c}\right|$ occurring in a short orbit arc. Over most of the plasmasphere, the Lorentz force has thus a negligible effect on SC dynamics. Nonetheless, the tether can generate power for local use over intermediate orbit segments, saving fuel-cell power for the regions outside the denser parts of plasmasphere and torus. From (8), one can find the average power along an orbit; for capture $(e=1)$ and negligible ohmic effects (small $\Lambda$ ), one finds

$$
\begin{aligned}
\frac{|\langle\dot{W}\rangle|}{m_{t}}= & \frac{0.2 N_{s}}{\rho_{t} h} \sqrt{\frac{\left(e v_{s 1} B_{s} L\right)^{3}}{m_{e}}}\left(\frac{a_{s}}{r}\right)^{9 / 2} \frac{N_{e}}{N_{s}} \\
& \times \frac{\left|\widetilde{r}_{M}-\widetilde{r}\right|}{2^{1 / 4} \widetilde{r}^{3 / 4} \sqrt[4]{\widetilde{r}_{M}^{2}-2 \widetilde{r}_{M} \widetilde{r}+\widetilde{r}^{3}}} \\
\approx & 0.884 \frac{\widetilde{r}_{M}^{3}}{\widetilde{r}^{21 / 4}} \frac{\left|\widetilde{r}_{M}-\widetilde{r}\right|}{\left(\widetilde{r}_{M}^{2}-2 \widetilde{r}_{M} \widetilde{r}+\widetilde{r}^{3}\right)^{1 / 4}} \frac{N_{e}}{N_{s}}\left(\widetilde{r}, \widetilde{r}_{M}\right) \frac{\mathrm{kW}}{\mathrm{kg}}
\end{aligned}
$$

where values of $L=50 \mathrm{~km}$ and $h=0.05 \mathrm{~mm}$ were used. This local power per unit tether mass is shown in Fig. 3.

Note that the results in Fig. 3 correspond to zero load impedance. Using the tether efficiently in a generator mode over intermediate orbit segments, with current along with the hollow cathodes on, requires a load impedance comparable to the impedance of OML current collection. Although the Lorentz power taken from the SC motion would be very small (when compared with the power produced during high-current operations, thus having negligible effect on orbit dynamics), the load would take a fraction (the generator efficiency $\eta_{g}$ ) of order unity of such power. Power generation during deorbiting was analyzed in [8]. For negligible ohmic effects, one finds the 


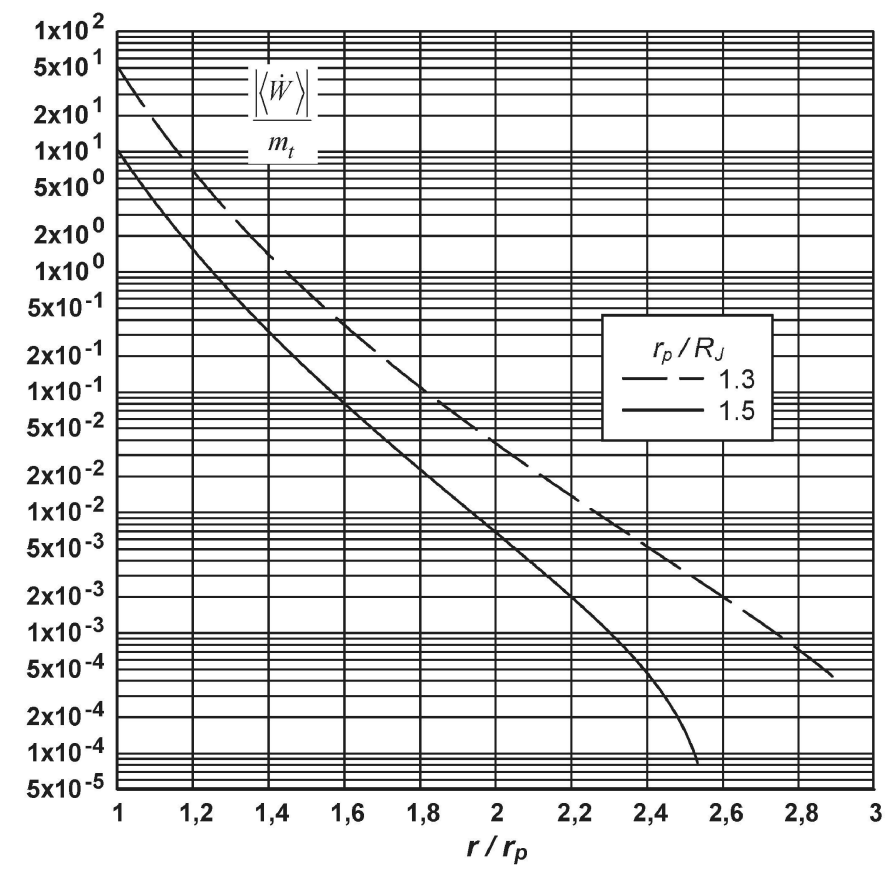

Fig. 3. Lorentz-drag power per unit tether mass in kilowatts per kilogram units versus position along the $(e=1)$ orbit of capture, within the plasmasphere $\left(r<3.8 R_{J}\right)$, for two values of perijove radius and the tape in Fig. 2.

following: 1) $\eta_{g}=10 / 19$ for the conditions of maximum load power, and 2) the Lorentz power itself is smaller than its value for no load impedance by a factor of $\sqrt{3 / 5} \times 19 / 15 \approx 0.589$. The load power attained under optimal conditions would then be less than as shown in Fig. 3 by a factor of $0.589 \times 10 / 19=$ 0.31 [8]. Yet, a maximum power per unit tether mass of $0.31 \times 5 \mathrm{~W} / \mathrm{kg}$ or about $300 \mathrm{~W}$ might be attained with the 202-kg 3-cm-wide reference tape, as far as $r=2.4 r_{p}=$ $3.12 R_{J}$ for the $1.3 R_{J}$ perijove.

\section{RADiation Dose}

As regards radiation, there exist two basic modifications of the $\mathrm{D} / \mathrm{G}$ model, which had originally covered the magnetic shell range of $1.09<L<16$. Later analysis of data from the Galileo Energetic Particle Detector led to modifications over the range of $8<L<16$ and the development of the so-called Galileo Interim Radiation Electron (GIRE) model [5]. GIRE somewhat reduces the dose rate, as compared with the D/G model, near the Europa and Ganymede orbits but leaves the $L<8$ range (dominant as regards radiation) unmodified and thus has only a moderate relative effect on the dose per orbit for orbits that reach very close to Jupiter. A second modification of the D/G radiation model covers the $L<4$ range, well inside the inner magnetosphere. It was based on a recent analysis that fitted the synchrotron emission data from Earth-based measurements. It primarily affects relativistic (multi-MeV) electron energies and the electron flux only in the narrow range of $2<L<2.3$ and thus will be ignored here [9].

A simple benchmark for estimating radiation effects over the orbit evolution of the tethered SC is the calculation of dose over a parabolic capture orbit. Calculations were carried out starting at $15 R_{J}$, moving inward to perijove, and ending back at $15 R_{J}$,

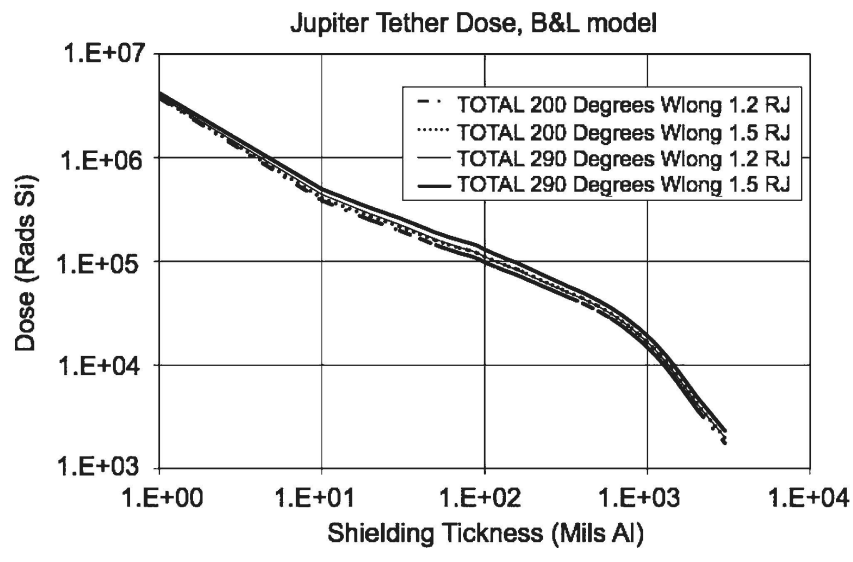

Fig. 4. Total dose-depth curves for an equatorial and parabolic orbit of capture for perijoves at two radii and two west longitudes (R. W. Evans).

using the GIRE radiation model. Fig. 4 shows the dose/depth curves for both $1.2 R_{J}$ and $1.5 R_{J}$ capture perijoves at $200^{\circ}$ and $290^{\circ}$ west longitudes in standard SIII coordinates (roughly corresponding to the minimum and maximum of dose).

Dose involves both fluence and the stopping power by a specified shielding material, typically aluminum; for any given shield thickness, incident particles below some energy will not come out at the opposite side of the shield. As a result, radiation dose, in terms of a reference material (silicon) placed behind the shield, will decrease with increasing shield thickness. A standard shielding configuration was used in the calculation of radiation dose, with the generic code involving an aluminum spherical shell for all $4 \pi \mathrm{sr}$.

Fig. 4 shows that dose is weakly dependent on longitude, reflecting the low values of both tilt and offset of the dipole describing the magnetic field in the inner magnetosphere (ignored in our analysis of both capture and orbit evolution). Independently, at distances very close to Jupiter, dose decreases, although weakly, as the perijove is located closer and closer to the planet. Full dose over the orbit capture is about 50-krad Si for 10-mm (or about 400-mil) Al shield thickness. It appears generally accepted that the electronic equipment to be used in future Jovian missions will need to be hardened well over 1-Mrad $\mathrm{Si}$; up to $10 \mathrm{~mm}$ of $\mathrm{Al}$ might be required, depending on the specific orbit.

If one proceeds along a sequence of orbits of decreasing apojove, comparable values of dose per orbit result. Fig. 5 shows the dose increment per orbit, for two perijove values, versus eccentricity (or, equivalently, apojove); the dose increment first increases and then exhibits a substantial decrease as $e$ is reduced.

\section{JOVIAN MOON TOUR}

The SC apojove can be lowered to any moon orbit, with a particular perijove allowing for resonance between the (elliptical) SC and the (circular) moon orbits. This would allow tangential conveniently slow flybys of the moon. More than one perijove pass per flyby would take place for such flybys, however; we find that this rapidly increases the accumulated radiation dose. We are thus considering here nontangential flybys at $1: 1$ resonance, with one perijove pass per flyby. The $1.3 R_{J}$-perijove 


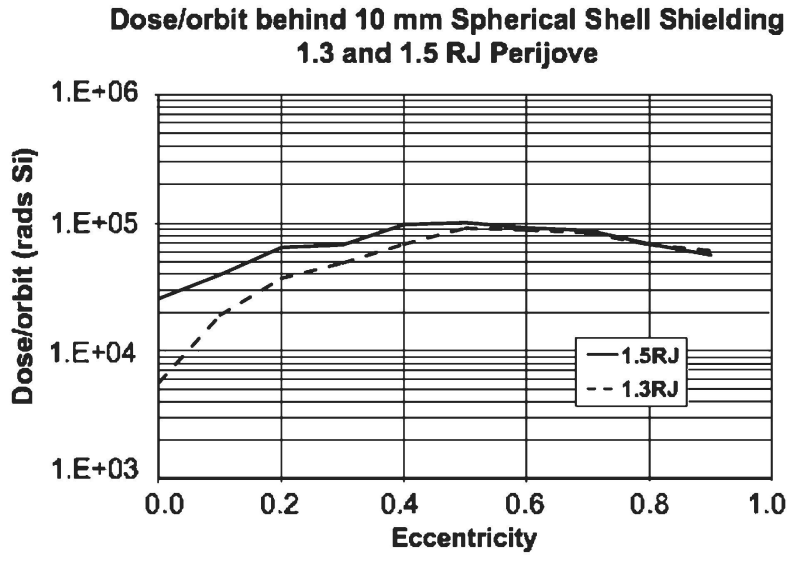

Fig. 5. Radiation dose per orbit versus orbital eccentricity for two perijove values and 10-mm Al shield thickness (R. W. Evans).

elliptical orbits at $1: 1$ resonances with the moons Ganymede, Europa, and $I o$, which lie at distances 15.0, 9.4, and 5.9R $R_{J}$, have eccentricities of $0.913,0.862$, and 0.779 , respectively.

Fig. 5 shows the radiation dose per perijove pass, at $1.3 R_{J}$, lying in the range of $5-7 \times 10^{4}$-rad $\mathrm{Si}$ for $10-\mathrm{mm}$ Al shield thickness, in the eccentricity range of $0.78-1$. Taking an approximate average of $6 \times 10^{4} \mathrm{rad}$ and keeping below a maximum accumulated dose of 3-Mrad Si allow up to 50 perijove passes. On the other hand, the sequence of orbits corresponding to a uniform eccentricity decrement $\Delta e=0.04$ allows one to reach the $1: 1$ resonances for the different moons very rapidly. This will make for a high number of flybys. Note that the last eccentricity decrement previous to any particular resonance must be reached in two convenient steps by switching the current off appropriately over part of the drag arc to allow for a first flyby of the respective moon. Switching off the current afterward over the entire resonance orbit would allow for repeated flybys, with the moon overtaking, each time, the slower moving SC.

We note that the large $J 2$ zonal harmonic coefficient in Jupiter's gravitational potential (0.01473 as against 0.00108 for the Earth) would have a minute but cumulative effect on the sequence of flybys for each moon. The apsidal precessions (once the nodal regressions are taken into account) for moon and flyby orbits differ in about $1.25^{\circ}$ per orbit for all three Ganymede, Europa, and Io cases. This will require slightly larger flyby orbits at 1.0035:1 resonances. Fig. 6 shows a schematic of Europa's flybys showing three well-separated orbits (i.e., the $1 \mathrm{st}, 6 \mathrm{th}$, and 11th). The moon is encountered at successively delayed times because of the differential apsidal precession that is compensated for by the slightly increased orbital resonance, as noted previously.

A tentative moon tour would involve 4 Ganymede, 20 Europa, and 16 Io flybys, for a total of 40 flybys. At an average of $6 \times 10^{4}$-rad Si per flyby, it makes a total of 2.4 Mrad. In addition, two perijove passes after capture are required prior to the pass leading to the first Ganymede flyby; one and two perijove passes are required prior to passes leading to the first Europa and Io flybys, respectively. This makes a total of 0.36-Mrad Si, with the combined accumulated dose being less than 3 Mrad.

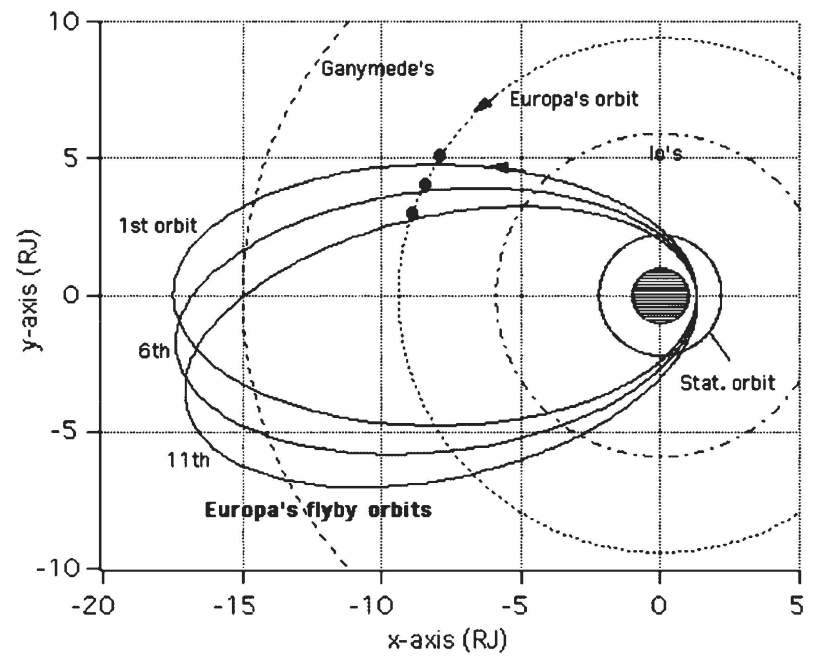

Fig. 6. Schematic of flyby orbits of Europa showing three (well-separated) encounters at orbits 1,6 , and 11 . The ratio of the periods of the flyby to Europa's orbit is $1.0035: 1$ to account for the differential nodal precession between the $\mathrm{SC}$ and the moon.

Power needs must also be considered in limiting the number of flybys; as suggested in Section III, the energy stored during the move down to each moon might allow for $120 \mathrm{~h}$ at $250 \mathrm{~W}$ for the 3-cm-wide tape and 650-kg SC. Because the Europa flybys would be of the most critical interest, we set its number greater than the number of flybys for Io, even though Io's orbital period is half the Europa period; this means that the energy stored would be shared over a larger lapse of time in visiting Europa. A total of $120 \mathrm{~h}$ over 20 visits would still allow $6 \mathrm{~h}$ per visit. We note that the primary acquisition of science data in the Juno mission will occur over a 6-h period centered at perijove, although science data from the just-completed perijove pass will be transmitted outside this window [10].

The total duration of the mission is quite short. The three apojove lowering stages, from capture to Ganymede resonance, and later to Europa and to Io resonances, would take 86.8, 5.0, and 4.6 days, respectively, for a total of less than 14 weeks. In turn, each Io flyby takes the Io period or about 1.77 days. Flybys for Europa and Ganymede take twice and four times as much, respectively. All flyby phases would then add to a total of about 18 weeks, with the entire duration of the mission being just over seven months. The extremely frequent access of the tethered $\mathrm{SC}$ to the orbits of Galilean moons, reducing any time-variation effect on measurements, is to be compared to the frequency of visits in the Galileo mission. Galileo made 34 close encounters or flybys in almost eight years; it thus took nearly three months on the average from one visit to the next. The price paid here is the rapid accumulation of radiation dose due to the tethered SC orbiting through the intense radiation belts near Jupiter on each moon visit.

\section{CONCLUSION}

We have analyzed an electrodynamic bare-tether mission at Jupiter, following the capture of an SC into an equatorial highly elliptical orbit with perijove at about 1.3 times the Jovian radius. The tethered $\mathrm{SC}$ can then rapidly and frequently visit 
Galilean moons. Repeated applications of the propellantless Lorentz drag on the (spinning) tether, at the perijove vicinity, can progressively lower the apojove at constant perijove, for a tour of moons.

A reinforced Al tape tether, which is $50 \mathrm{~km}$ long and $0.05 \mathrm{~mm}$ thick, coated for 0.8 thermal emittance and spinning with about 12-min period, can capture an SC with full mass over three times its mass, taking the 1.02 hyperbolic eccentricity of a Hohmann transfer down to a first elliptical 0.98 eccentricity orbit. No characteristic dimensionless number involves the tape width. The SC mass just scales up with width in a range easily allowing from about 0.2 to $5 \mathrm{t}$. A 3-cm-wide tape, with its mass being $202 \mathrm{~kg}$ and the full $\mathrm{SC}$ mass being about $650 \mathrm{~kg}$, was taken as the reference tether for some numerical considerations.

Electrical energy is generated and stored as the SC moves from an orbit at $1: 1$ resonance with a moon, down to resonance with the next moon. Switching tether current off, stored energy, allowing $120 \mathrm{~h}$ at $250-\mathrm{W}$ power for the $3-\mathrm{cm}$-wide tape and $650-\mathrm{kg} \mathrm{SC}$, is then used as the SC makes a number of flybys of each moon, tentatively 4, 20, and 16 for Ganymede, Europa, and $I o$, respectively. Because Europa flybys would be of the most critical interest, we set its number greater than the number of flybys for Io, even though Io's orbital period is half the Europa period; this means that the energy stored would br shared over a larger lapse of time in visiting Europa. A tota of $120 \mathrm{~h}$ over 20 visits would still allow $6 \mathrm{~h}$ per visit.

Radiation dose is calculated throughout the mission, during capture, flybys and moves between moons. The tour mission is limited by both power needs and accumulated dose. The threestage apojove lowering down to Ganymede, Io, and Europa resonances would total less than 14 weeks, while the Ganymede, Europa, and $I o$ flybys would add up to 18 weeks, with the entire mission taking just over seven months and the accumulated radiation dose keeping under $3 \mathrm{Mrad}(\mathrm{Si})$ at $10-\mathrm{mm} \mathrm{Al}$ shield thickness.

\section{REFERENCES}

[1] J. R. Sanmartin and E. C. Lorenzini, "Exploration of outer planets using tethers for power and propulsion," J. Propuls. Power, vol. 21, no. 3, pp. 573-576, 2005.

[2] J. R. Sanmartin, M. Charro, E. C. Lorenzini, H. B. Garrett, C. Bramanti, and C. Bombardelli, "Electrodynamic tether at Jupiter-I: Capture operation and constraints," IEEE Trans. Plasma Sci., vol. 36, no. 5, pp. $2450-$ 2458 , Oct. 2008
[3] F. Bagenal, T. Dowling, and W. McKinnon, Eds., Jupiter, the Planet, Satellite, and Magnetosphere. Cambridge, U.K.: Cambridge Univ. Press, 2004. Table 24.1.

[4] N. Divine and H. B. Garrett, "Charged particle distributions in Jupiter's magnetosphere," J. Geophys. Res., vol. 88, no. A9, pp. 6889-6903, Sep. 1983.

[5] H. B. Garrett, I. Jun, J. M. Ratliff, R. W. Evans, G. A. Clough, and R. W. McEntire, Galileo Interim Radiation Electron Model, Jet Propuls. Lab., Pasadena, CA, Publication 03-006, 2003.

[6] F. Barbir, L. Dalton, and T. Molter, "Regenerative fuel cells for energy storage: Efficiency and weight trade-offs," in Proc. 1st Int. Energy Convers. Energy Conf., Portsmouth, VA, Aug. 17-21, 2003.

[7] C. P. García, B.-J. Chang, D. W. Johnson, D. J. Bents, V. J. Scullin, and I. J. Jakupca, "Round trip energy efficiency of NASA Glenn regenerative fuel cell system," John H. Glenn Res. Center, Cleveland, OH, NASA/TM 2006-214054, 2006.

[8] J. R. Sanmartin, M. Martinez-Sanchez, and E. Ahedo, "Bare wire anodes for electrodynamic tethers," J. Propuls. Power, vol. 9, no. 3, pp. 353-360, 1993.

[9] H. B. Garrett, S. M. Levin, S. J. Bolton, R. W. Evans, and B. Bhattacharya, "A revised model of Jupiter's inner electron belts: Updating the Divine radiation model," Geophys. Res. Lett., vol. 32, no. 4, pp. L04 104-1L04 104-5, 2005.

[10] R. Grammier, "An overview of the Juno mission to Jupiter," in Proc. Int. Symp. Space Technol. Sci., Kanazawa, Japan, Jun. 4-11, 2006. 